\section{NK cell action at a distance}

NKG2D is an activating receptor on NK cells that recognizes a variety of ligands and is involved in the immune response to tumors. In Science, Raulet and colleagues investigate the role of a secreted form of the NKG2D ligand MULT1 in tumor rejection. Tumors secrete such NKG2D ligands, and generally these are thought to antagonize the activation of NK cells. Here, however, the authors find that secreted MULT1 improves the recognition and destruction of implanted tumors by NK cells. Normally, the interaction of NKG2D with membrane-bound ligands found on cells in the tumor microenvironment results in downregulation of NKG2D and desensitization of NK cells. However, in the presence of high-affinity monomeric secreted MULT1, NKG2D is blocked from interacting with other membrane-bound NKG2D ligands and the receptor therefore remains in large abundance. These findings overturn previous hypotheses about how secreted ligands can affect immune responses.

ZF

\section{Rare protein modification}

Arginine methylation is an epigenetic histone modification that affects transcription, but the role of this post-translational modification in cellular functions remains unclear. In Nature Communications, Acuto and colleagues use isomethionine methyl-SILAC to identify arginine-methylation sites in human peripheral blood $\mathrm{T}$ cells. Approximately 1,200 proteins linked to transcription, chromatin remodeling, cytoskeleton remodeling and protein transport, cell metabolism and signaling networks undergo arginine methylation in T cells. Arginine methylation is found on adaptors, kinases and phosphatases linked to proximal TCR signaling, such as SLP-76, PLC- $\gamma 1$, SHIP-1, SHP-2 and PI(3)K, and on key transcription factors that regulate $\mathrm{T}$ cell fate programs, such as Notch1, Foxp3, Bcl-11b, IRF4, T-bet and NF- $\kappa$ B. The activation of T cells induces changes in the methylation stoichiometry in $10 \%$ of the arginine-methylated peptides, mostly in proteins involved in mRNA splicing, which suggests that arginine methylation can be dynamic.

Nat. Commun. (7 April 2015) doi:10.1038/ncomms7758

\section{Strengthening barriers}

Siglecs are a family of sialic acid-binding receptors with generally immunoinhibitory functions. In eLife, Gagneux et al. investigate whether the substantial variability in Siglec members in both expression and type contributes to the differences between species in aging. In many, varied mammalian species, the authors find a positive correlation between the number of Siglec-encoding genes and species lifespan. Furthermore, mice deficient in one member, Siglec-E, have a significantly shorter lifespan. Siglec-E-deficient mice have no overt alteration in phenotype other than accelerated cognitive and physical decline. Siglec-E is expressed mainly by phagocytes, and Siglec-E-deficient mice have enhanced production of reactive oxygen species (ROS) and immunological activation in the liver. This enhancement in ROS is correlated with increased signs of DNA damage, as well as oxidation of lipids and proteins, classic signs of aging. These data suggest that if it is not 'buffered' appropriately, activation of the innate immune system may lead at least in part to more rapid aging.

eLife (7 April 2015) doi:10.7554/eLife.06184

\section{Tissue-specific surveillance}

The adhesion of $\mathrm{CD}^{+} \mathrm{T}$ cells to the fenestrated endothelium barrier in the liver sinusoids may differ from their usual pattern of rolling, adhesion and extravasation in other tissues. In Cell, Guidotti et al. use a model of hepatitis B virus infection in mice to show that homing of $\mathrm{CD}^{+} \mathrm{T}$ cells to the liver is independent of selectins, integrins, $\mathbf{G a}_{i}$ protein-coupled chemokine receptors and antigen recognition. Instead, $\mathrm{CD}^{+} \mathrm{T}$ cells use hyaluronan to dock on small islands of $\mathrm{CD} 44^{+}$platelet aggregates that adhere to the liver sinusoid epithelial cells. $C D 8^{+} \mathrm{T}$ cells crawl along liver sinusoids and extend cellular protrusions through the endothelial fenestrae to probe the underlying hepatocytes, and recognition of antigen leads to activation of $\mathrm{CD} 8^{+} \mathrm{T}$ cells and cytokine production before extravasation. Liver fibrosis diminishes sinusoid fenestration, which suggests that it might also impair the immunosurveillance of infected or transformed hepatocytes by $\mathrm{T}$ cells.

Cell (23 April 2015) doi:10.1016/j.cell.2015.03.005

Written by Laurie A. Dempsey, Zoltan Fehervari \& Ioana Visan
West Nile virus is a mosquito-borne virus that can cause severe neurological disease or death in susceptible individuals. In Science Translational Medicine, Lazear et al. report that IFN- $\lambda$ lessens neurologic sequelae in infected mice by enhancing bloodbrain-barrier function and restricting entry of the virus into the central nervous system (CNS). Mice that lack the IFN- $\lambda$ receptor IFNLR1 harbor more virus in the CNS after infection with West Nile virus. IFN- $\lambda$ does not elicit a cell-intrinsic effect in antiviral responses; instead, it acts on brain microvascular endothelial cells to enhance colocalization of the junction proteins claudin-5 and ZO-1 and thereby makes the blood-brain barrier less permeable to blood-borne viruses. Surprisingly, this response does not require signaling via STAT1 or protein synthesis. Nevertheless, these findings highlight a means for modulating CNS permeability by IFN- $\lambda$.

Sci. Transl. Med. 7, 284ra59 (2015)

\section{Targeting leukemic stem cells}

The resistance of leukemic stem cells to conventional therapies poses challenges to the long-term cure of various hematopoietic malignancies. In Nature Cell Biology, Kang et al. identify the surface receptor LAIR1 (which contains immunoreceptor tyrosine-based inhibitory motifs) as a potential therapeutic target expressed on leukemic stem cells. Many acute leukemia cell lines express LAIR1, but knockdown of LAIR1 via short hairpin RNA diminishes the viability of such cells in vitro and upon serial transplantation into mice; however, loss of LAIR1 expression does alter normal hematopoiesis. Collagenmediated crosslinking of LAIR1 promotes its interaction with the phosphatase SHP-1, which acts as a scaffold to recruit the kinase CAMK1. This signaling axis activates the transcription factor CREB that enhances survival of leukemic stem cells. Interfering with this axis increases apoptosis of leukemic stem cells, which makes LAIR1 a potential target for therapeutic intervention.

$L A D$ Nat. Cell Biol. (27 April 2015) doi:10.1038/ncb3158 